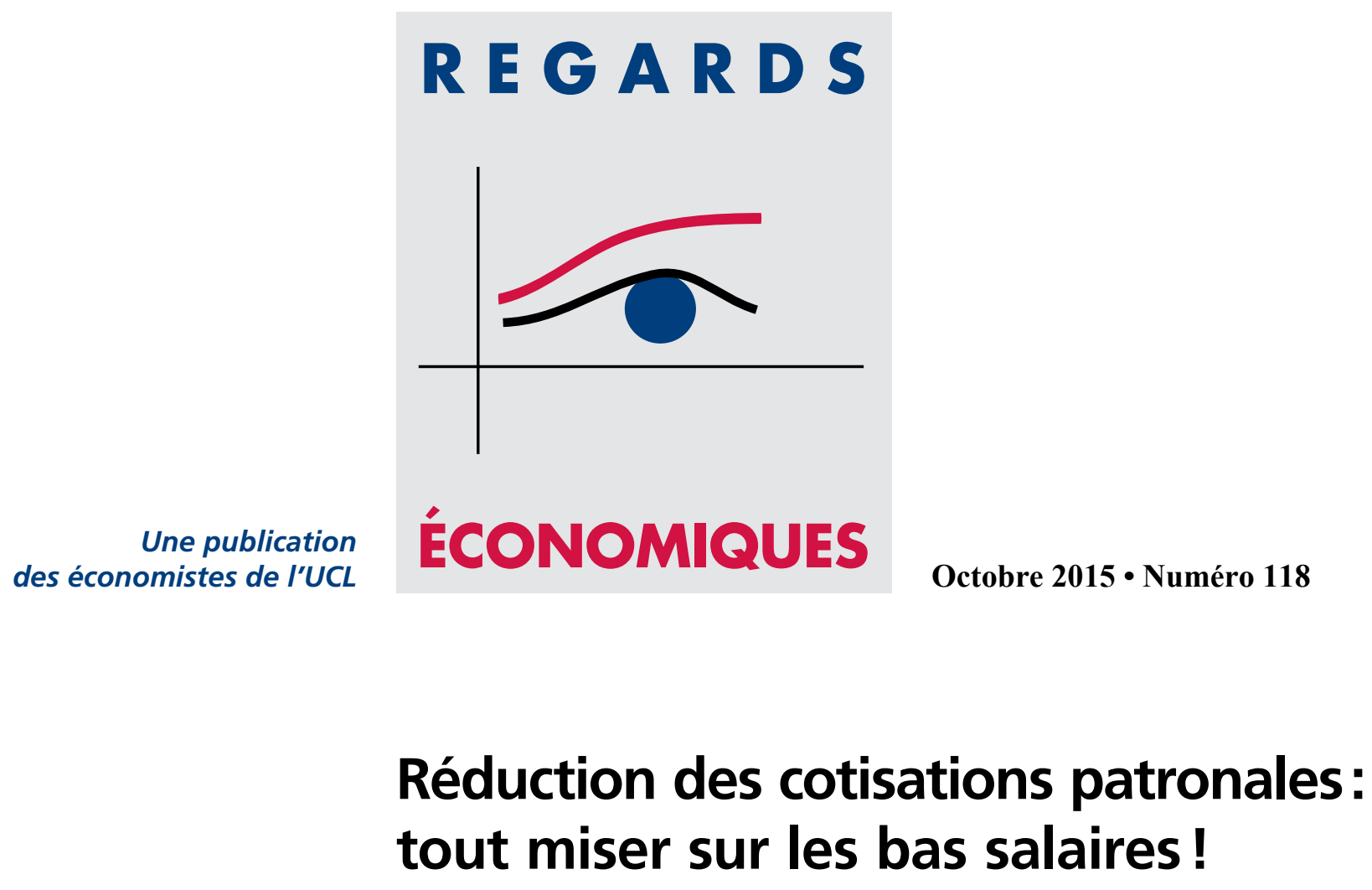

Une réduction des cotisations patronales ne peut être efficace que si elle est ciblée sur les travailleurs dont le coût salarial est un élément déterminant de la stratégie d'embauche et de licenciement des entreprises et si elle vise des emplois pour lesquels il n'y a pas de pénurie de main d'ouvre. Ces conditions ne peuvent être remplies que pour les emplois qui requièrent un faible niveau de compétences. L'enveloppe budgétaire prévue pour les réductions de charges en 2016 pourrait à terme engendrer un supplément de 28.000 à 47.000 emplois si les recommandations de ce numéro de Regards économiques sont suivies.

\section{Muriel Dejemeppe}

Bruno Van der Linden*

Le «pacte de compétitivité» établi sous le gouvernement fédéral précédent (le gouvernement Di Rupo) prévoyait un renforcement de la réduction structurelle de cotisations sociales patronales ${ }^{1}$ étalé dans le temps. Dans le prolongement de son accord de gouvernement, l'équipe fédérale actuelle a rappelé en juillet dernier sa volonté de diminuer les charges patronales dès 2016. L'objectif affiché est d'atteindre un taux de cotisations patronales de $25 \%$, mais les modalités précises de mise en œuvre de cette réforme sont en discussion.

Le niveau du taux de cotisations patronales «facial» (c'est-à-dire abstraction faite de toute forme de réduction et de subvention) dépend de nombreuses caractéristiques. ${ }^{2}$ Il s'élève par exemple à $32,9 \%$ pour les travailleurs de statut employé au sein d'entreprises de 1 à 9 travailleurs. A partir de 20 travailleurs, ce taux s'élève à $34,7 \%$. Toutefois, la réglementation prévoit que la réduction structurelle des cotisations patronales ne peut dépasser $32,4 \%$ du salaire brut, ce qui signifie qu'une réduction complète des charges patronales de sécurité sociale n'est pas autorisée. Ce taux de $32,4 \%$ sera donc notre point de référence.

Ce taux facial est trompeur car il existe une variété de formes de réduction des cotisations patronales ${ }^{3}$ qui amènent le taux effectif de cotisations patronales souvent bien en deçà de son niveau facial. L'objectif du gouvernement fédéral est d'opérer une clarification de telle sorte que le taux facial soit à tout le moins plus informatif de la réalité. Apporter de la clarté est un motif en soi louable car l'accumulation des mécanismes d'allègement est source de confusion. Mais cette clarification ne suffit pas à justifier la mesure. La justification affichée est l'amélioration de nos performances en matière d'emploi. Or, comme nous l'expliquons ci-dessous,

\footnotetext{
${ }^{*}$ Les auteurs remercient Vincent Bodart pour ses commentaires sur une version préliminaire du texte.

${ }^{1}$ Les principes et modalités d'application actuels de la réduction structurelle sont détaillés sur http://www.emploi.belgique.be/defaultTab.aspx?id=5336.

${ }^{2}$ Voir par exemple http://vbo-feb.be/fr-be/News--Media/Faits--statistiques-/Cotisations-sociales--Premier-trimestre-2015/.

${ }^{3}$ Voir le tableau 6-9 du Conseil Central de l'Economie (2015). Pour plus de détails, voir

https://www.socialsecurity.be/employer/instructions/dmfa/fr/latest/instructions/deductions/introduction.html.
} 
l'ampleur de cette amélioration dépendra étroitement des modalités précises de mise en œuvre de la réforme envisagée. Dans ce numéro de Regards économiques, nous nous concentrons donc sur cet aspect.

Alors que les modalités d'un allègement renforcé des charges patronales sont en discussion au niveau fédéral, les Régions définissent en ce moment leurs priorités concernant le transfert des mesures fédérales de soutien financier à l'emploi lié à la sixième réforme de l'Etat (réductions de cotisations patronales sur les «groupes cibles ONSS» et «l'activation des allocations de chômage»). Nous estimons que leurs décisions actuelles et futures seront conditionnelles aux orientations prises par le gouvernement fédéral en matière d'allègement du coût du travail. L'importance de ces décisions régionales n'échappe à personne mais elles n'ont pas fait l'objet de cette analyse.

\section{La réduction structurelle des cotisations patronales : situation actuelle}

La Belgique se caractérise par une fiscalité et une parafiscalité comparativement lourdes sur le travail salarié. Le taux d'imposition implicite sur le travail salarié s'élevait en effet en 2012 à 42,8 \% en Belgique, un niveau qui dépasse celui de nos voisins. ${ }^{4} \mathrm{Ce}$ constat déjà ancien est persistant malgré des efforts non négligeables pour alléger cette pression (para)fiscale. Sur base de calculs du Bureau Fédéral du Plan, le Conseil Central de l'Economie (2015) indique qu'en 2013, l'ensemble des réductions de cotisations sociales patronales s'élevaient à 5,1 milliards $€$, représentant 3,3\% de la masse salariale du secteur privé (tableau 6-11). Ce montant englobe la réduction dite structurelle et les réductions «groupes cibles» qui sont à présent régionalisées. Aux réductions de cotisations patronales s'ajoutent des subventions salariales octroyées par l'Etat fédéral, les Régions ou la sécurité sociale. ${ }^{5}$ Hors mesures spécifiques sectorielles, les subventions salariales via la fiscalité fédérale représentent 2,6 milliards $€$, soit $1,7 \%$ de la masse salariale du secteur privé (tableau 6-11).

La mesure de réduction structurelle des cotisations sociales patronales, qui demeure une compétence fédérale, est applicable à la majorité des travailleurs salariés du secteur privé (plus de deux millions d'équivalents temps plein). Si l'on se limite à la catégorie générale ${ }^{6}$, l'allègement de cotisations existant est formé d'un forfait, qui est majoré dans le cas de travailleurs à bas salaires et dans celui de travailleurs à salaires élevés. La figure 1 décrit le niveau mensuel de la réduction structurelle de cotisations patronales en fonction du niveau de salaire brut mensuel au $3^{\mathrm{e}}$ trimestre de 2015. La figure 2 présente le taux facial de cotisations patronales de référence $(32,4 \%)$ et, en vert, le taux effectif du fait de la réduction structurelle. L'objectif d'un taux de $25 \%$ est aussi représenté. Le taux de cotisations patronales effectif s'élève à 19,5\% au niveau du Revenu Minimum Moyen Mensuel Garanti (RMMMG), soit au $1^{\text {er }}$ janvier 2015 la somme de $1.559 €$ bruts/mois si le travailleur a 21 ans. Le taux effectif s'élève actuellement à $25 \%$ lorsque le salaire brut avoisine $2.090 € /$ mois. Au-delà, l'écart entre le taux de cotisations patronales actuel effectif et le taux de cotisations de $25 \%$ grandit pour atteindre un maximum de 4 points de pourcentage au niveau de salaire brut de $4.470 € /$ mois. Ensuite, cet écart se résorbe

\footnotetext{
${ }^{4}$ Conseil Central de l'Economie (2015), tableau 2-2. Le numérateur de ce taux implicite comprend l'impôt sur le revenu dû par les salariés augmenté de l'ensemble des cotisations sociales (personnelles et patronales) effectivement dues. Le dénominateur de ce taux mesure les salaires et traitements bruts. Pour plus de détails voir la note doc12-224 du Conseil Central de l'Economie : http://www.ccecrb.fgov.be/txt/fr/doc12-224.pdf.

${ }^{5}$ Exemption du paiement du précompte professionnel pour certaines catégories de travailleurs (travail d'équipe et de nuit, ...), activation des allocations de chômage, intervention de l'Etat dans les titres-services, etc. Pour une liste plus détaillée, voir le tableau 6-10 du Conseil Central de l'Economie (2015) ou la partie A du rapport de juillet 2013 du groupe d'experts «Compétitivité et Emploi» disponible à l'adresse : http://statbel.fgov.be/nl/binaries/OK_A4_Kaft_rapport_GECE_tcm325-228564.pdf.

${ }^{6}$ Par conséquent on ignore les travailleurs dont l'employeur relève du «Maribel social» ou est une «entreprise de travail adapté».
} 
cet écart se résorbe très progressivement. Par rapport au taux facial de référence $(32,4 \%)$, la réduction structurelle en vigueur fait passer le taux effectif moyen de charges patronales à $26 \%$. La figure 2 indique également que la plus forte densité de salariés se situe entre environ 1.760 et $2.740 €$ bruts/mois (voir courbe en noir).

Figure 1. Montant mensuel de la réduction structurelle de cotisations sociales patronales

(travailleurs de la catégorie générale) au $3^{\mathrm{e}}$ trimestre de 2015

en fonction du salaire mensuel brut (entre le RMMMG à 21 ans et $6000 € /$ mois)

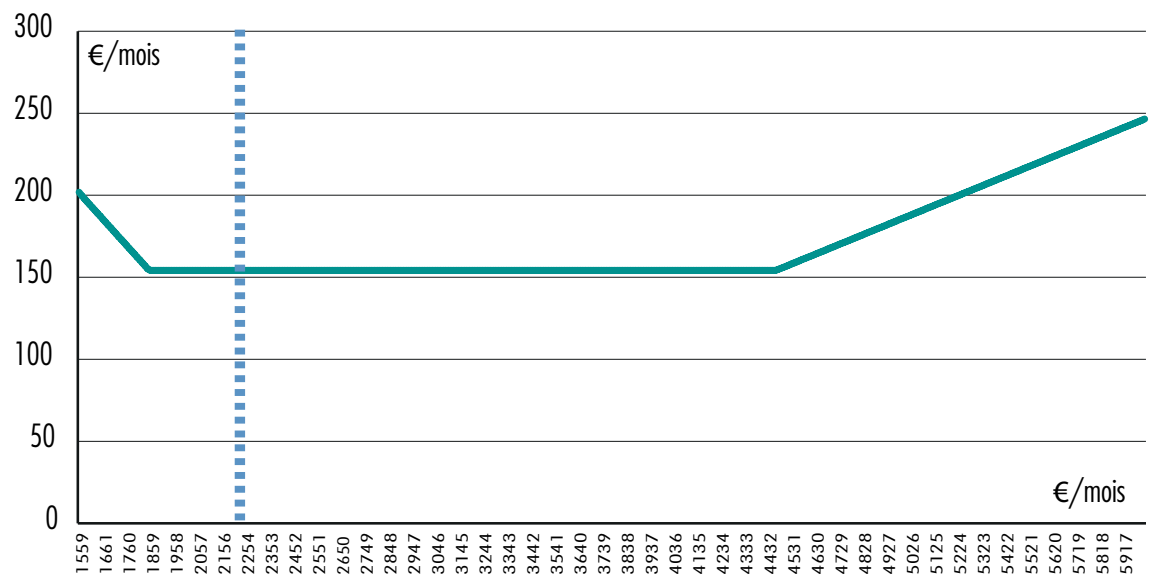

Figure 2. Le taux de cotisations patronales $(\%)$ au $3^{\mathrm{e}}$ trimestre de 2015 après prise en compte de la réduction structurelle (échelle de gauche) et le taux de fréquence (\%) des salariés (échelle de droite) en fonction du salaire mensuel brut (entre le RMMMG à 21 ans et $6000 € /$ mois)

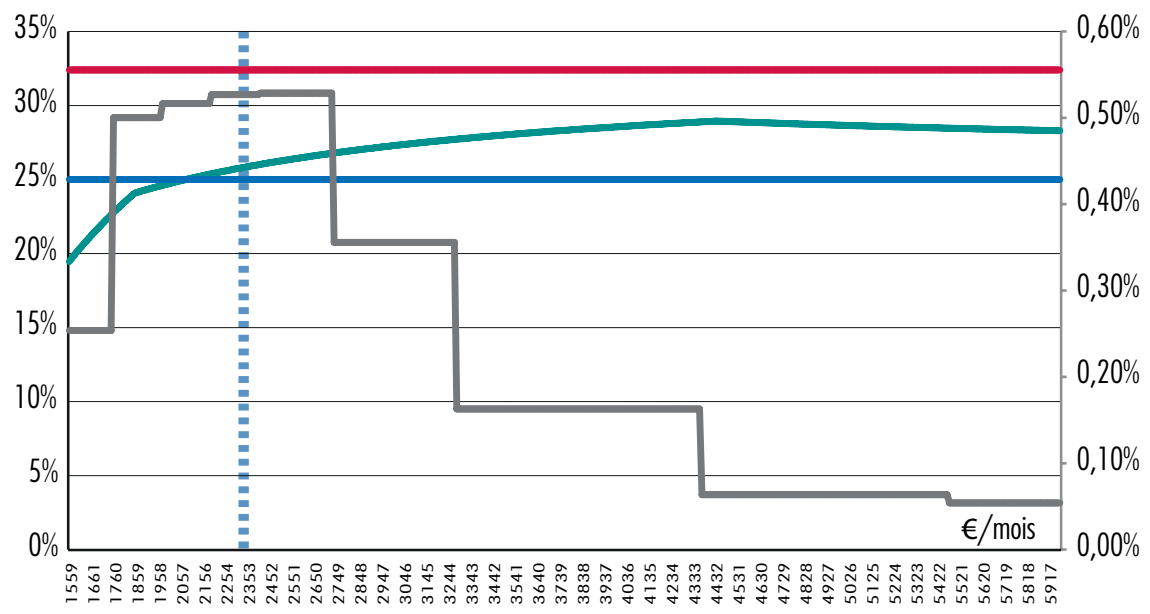

Note de lecture : Le taux effectif de cotisations (courbe verte) est calculé en déduisant la réduction structurelle du montant facial de cotisations patronales sous l'hypothèse d'un taux facial de $32,4 \%$, la différence étant divisée par le salaire brut. Comme nous l'avons rappelé en introduction, le taux facial est en réalité supérieur à $32,4 \%$. Le taux effectif que nous calculons est donc une sous-estimation du taux effectif réel. La courbe noire représente une approximation du taux de fréquence des salariés (ONSS secteur privé) en équivalents temps-plein (ETP $)^{7}$ en fonction du salaire brut pour les travailleurs âgés de 18 ans au moins. Ce taux de fréquence est le rapport entre le nombre de salariés ETP dont le salaire brut/mois est compris entre $\mathrm{X} €$ et $\mathrm{Y} €$, et le nombre total de salariés ETP. La ligne horizontale rouge représentant le taux facial de $32,4 \%$; la ligne horizontale bleue représente le taux de $25 \%$, objectif du gouvernement fédéral. La ligne verticale en pointillé se situe à 1,5 fois le RMMMG.

\footnotetext{
${ }^{7}$ Comme il est indiqué sur http://www.rsz.fgov.be/fr/statistiques/faq, le calcul du volume de travail en ETP généré par une prestation de travail est le produit de deux facteurs :

(i) d'une part, le rapport entre les prestations de travail réelles (périodes rémunérées et de vacances) du travailleur et le maximum possible des prestations de travail;

(ii) d'autre part (pour les travailleurs à temps partiel), le rapport entre le nombre moyen d'heures de travail par jour de la prestation et le nombre d'heures de travail par jour de la personne de référence.
} 


\section{Le projet du gouvernement fédéral}

Nous nous limitons strictement aux moyens disponibles pour la réduction des cotisations patronales dans le secteur privé et nous n'incorporons pas les dépenses en faveur des indépendants et des très petites entreprises ni celles destinées à renforcer les dispenses de précompte professionnel en cas de travail en équipe et en continu. De même, dans les limites de ce texte, nous ne traitons pas des moyens visant à relever les rémunérations nettes des travailleurs.

Les décisions prises lors du conclave budgétaire de juillet 2015 portent sur des mesures nouvelles et sur un réaménagement des mesures de réduction du coût du travail prévues dans la loi du 15 mai 2014 portant exécution du pacte de compétitivité, d'emploi et de relance (du gouvernement Di Rupo). Ensembles, les moyens alloués à la réduction structurelle des cotisations patronales dans le privé s'élèvent à 2.335 millions $€$ sur la période 2016 - 2019, soit 1.280 en 2016, 700 en 2018 et 355 en 2019.

Abstraction faite de la zone de «salaires élevés» où la réduction structurelle croît avec le salaire brut (voir figure 1), la formule de calcul de la réduction structurelle actuelle $R$ (€/trimestre) s'écrit schématiquement pour un temps-plein :

$$
\begin{aligned}
& \text { si } S<S_{0}: R=F+\alpha\left(S_{0}-S\right) \\
& \text { si } S \geq S_{0}: R=F
\end{aligned}
$$

où $S$ est le salaire brut et $S_{0}$ le seuil «bas salaire». Celui-ci est actuellement de $5.560,49 €$ (ou 1.853,50 €/mois, voir figure 1). Lorsque $S$ excède $S_{0}, R$ n'est autre que le forfait $F$ qui est actuellement de 462,6€ (ou 154,2 €/mois, voir figure 1). Lorsque $S$ est inférieur à $S_{0}$, le montant de la réduction est majoré de façon d'autant plus importante que le salaire se rapproche du RMMMG. Le pacte de compétitivité, d'emploi et de relance ne modifiait pas la pente $\alpha$. Elle valait et vaut toujours actuellement 0,162 . Ceci signifie que, pour les bas salaires, chaque euro supplémentaire de salaire brut entraîne une diminution de la réduction $R$ de 16 centimes (voir figure 1). Le pacte prévoyait en revanche trois relèvements successifs du forfait $F$ et du seuil $S_{0}$.

Le gouvernement fédéral actuel entend supprimer à terme le forfait $F$ (pour tous les niveaux de salaire) et le supplément d'allègement sur les «salaires élevés» afin que les économies ainsi réalisées contribuent au financement du passage du taux facial de cotisations actuel à l'objectif de $25 \%$. Comme on le voit à la figure 2 , sous environ $2.090 € /$ mois, le taux effectif de cotisations patronales actuel est déjà inférieur à $25 \%$. Sans pouvoir exclure avec certitude ce scénario, le gouvernement fédéral ne paraît pas avoir l'intention de ramener le taux de cotisations sur les bas salaires à cet objectif de $25 \%$. Le maintien dans l'enveloppe budgétaire d'une partie des moyens prévus dans le pacte de compétitivité laisse même penser qu'une part des 2.335 millions $€$ sera disponible pour une forme de renforcement des réductions de cotisations patronales sur les bas salaires.

La première question qu'on peut se poser est de savoir s'il est opportun d'amplifier encore les réductions structurelles du coût du travail en Belgique. En 2013, l'Etat fédéral consacrait près de $2 \%$ de son produit intérieur brut (395,3 milliards $€)$ à des réductions structurelles de cotisations patronales $(5,1$ milliards $€)$ et des subventions salariales (2,6 milliards $€$ ). Alors, stop ou encore ?

L'argument souvent avancé pour réduire les cotisations sociales patronales porte sur la sauvegarde de la compétitivité, sans nécessairement faire un lien étroit entre celle-ci et l'emploi. Le dernier rapport technique du Conseil Central de l'Economie indique qu'un écart de coût salarial se maintient par rapport à nos trois 
... Stop ou encore? pays voisins principaux. La prise en compte des subventions salariales complique certes la comparaison (voir l'exercice fouillé du Groupe d'experts «Compétitivité et Emploi» en juillet $2013^{8}$ ), mais révèle que cet écart est sans doute sur-estimé. Sous l'angle de la compétitivité, certains estiment donc qu'on est déjà allé assez loin en matière d'allègement du coût du travail.

Pour ceux qui considèrent que les cotisations sociales sont des primes d'assurance, une hausse des cotisations sociales n'aurait aucun effet sur le coût du travail car elle est compensée par une réduction du salaire net. Sous cet angle-là, toute diminution de la prime (sous la forme d'une réduction des cotisations sociales) devrait être rétrocédée aux travailleurs via un salaire net plus élevé. Le caractère obligatoire de la sécurité sociale, la présence de minima et de maxima dans les allocations sociales sans équivalent dans le niveau des cotisations payées, l'ouverture de certains droits à des personnes n'ayant jamais cotisé etc. peuvent au contraire conduire à la perception que les cotisations sociales s'apparentent davantage à une taxation du travail. Si c'est le cas, la manière dont les baisses de cotisations se répercutent sur le salaire net et sur le coût salarial devient plus complexe à déterminer. L'analyse économique produit certaines prédictions à ce sujet mais, au bout du compte, ces répercussions doivent être estimées à l'aide d'outils statistiques (économétriques) et sur base de données historiques. Ces outils seront évoqués à la section suivante.

Notre préoccupation est le maintien au fil des décennies d'un taux de chômage élevé des peu qualifiés, dans l'absolu en Wallonie et à Bruxelles, et par rapport à la moyenne régionale du taux de chômage en Flandre. Quelles que soient les améliorations hautement souhaitables des compétences de notre population, les travailleurs peu qualifiés sont là et le seront encore demain. Une absence de réponse à leur situation fréquente de non-emploi pose de nombreux problèmes à cette population et fait notamment le lit de tous les extrémismes politiques ${ }^{9}$. Comme nous le verrons ci-dessous, une réduction du coût du travail ciblée adéquatement sur les bas salaires contribue à apporter une réponse à cette préoccupation.

Mais soyons clairs : l'enveloppe budgétaire de 2.335 millions $€$ ne permettra pas de financer à la fois une réduction généralisée des cotisations patronales (le passage à un taux facial effectif de $25 \%$ ) et un renforcement significatif de la réduction structurelle sur les bas salaires (via un relèvement de $\alpha$ et/ou de $S_{0}$ ). D'ici 2019, des choix importants devront donc être faits quant à l'affectation de cette enveloppe budgétaire. Face à l'ampleur des fonds publics investis, on est en droit de s'attendre à ce que chaque euro qui sera investi dans une réduction supplémentaire de charges patronales d'ici à 2019 le soit avec la plus grande efficacité possible, c'est-à-dire en maximisant les effets sur l'emploi (de la meilleure qualité possible). Cet objectif est précisément le fil conducteur de notre analyse.

L'étude récente de Rycx et al. (2015) conclut que les entreprises industrielles et des services localisées en Belgique ont, sur la période 1999-2010, un différentiel entre la productivité du travail et son coût qui est défavorable à l'occupation de travailleurs peu qualifiés (en particulier s'ils sont jeunes). La plupart des études qui se sont intéressées à mesurer les effets des exonérations de charges patronales sur l'emploi en Belgique consistent à construire un modèle macroéconomique puis à y simuler l'effet de réformes (Bassilière et al., 2005, Stockman, 2007, Bureau fédéral du Plan et Banque nationale de Belgique, 2011). D'autres études utilisent des

\footnotetext{
${ }^{8}$ http://statbel.fgov.be/nl/binaries/OK_A4_Kaft_rapport_GECE_tcm325-228564.pdf.

${ }^{9}$ Dans le cas allemand, voir par exemple Falk et al. (2011).
} 


\section{... Encore mais comment?}

modèles d'équilibre général calculable (Sneessens, et al., 2005, Pierrard, 2005, Batyra et Sneessens, 2010)..$^{10}$ Enfin, plusieurs auteurs ont mesuré l'impact de mesures existantes à l'aide de méthodes d'évaluation économétriques (dans le cas belge, voir Cockx, 2005 et Goos, 2007). ${ }^{11}$ Les évaluations disponibles des effets de réductions structurelles de cotisations patronales en Belgique et à l'étranger convergent sur le premier constat suivant :

\section{Les réductions de cotisations patronales ciblées sur les bas salaires ont un effet positif sur l'emploi et la production nettement plus important que les réductions non ciblées (comme une réduction purement linéaire s'appliquant à tous les salariés).}

Les raisons de ce constat sont les suivantes. A tout moment du cycle économique, un nombre important d'entreprises envisage de créer de l'emploi et un autre nombre, lui aussi important, envisage d'en détruire. Les réductions de cotisations patronales ont pour but de stimuler la création d'emploi par les entreprises du premier groupe et à freiner la propension à détruire de l'emploi au sein du second groupe. Or, la quantité de main d'œuvre désirée par les entreprises est plus sensible au coût du travail parmi les travailleurs moins qualifiés. Cela s'explique par une productivité du travail généralement moindre alors que les coûts salariaux minimaux sont eux relativement élevés. Une autre explication est la plus grande aisance à remplacer de la main d'œuvre peu qualifiée par de l'équipement ou du travail réalisé dans des pays à bas salaires, ${ }^{12}$ voire par du travail non déclaré. La main d'œuvre de qualification moyenne est, il est vrai, elle aussi soumise aux menaces de l'automatisation et de la délocalisation, particulièrement lorsque les tâches exercées sont routinières. ${ }^{13}$ Les niveaux de salaires plus élevés de ces travailleurs rendent cependant très coûteuse une réduction structurelle substantielle des cotisations sociales patronales.

Les modèles du Bureau fédéral du Plan et de la Banque nationale de Belgique confirment que le ciblage sur les travailleurs à bas salaires a un impact bien plus important sur l'emploi qu'une mesure de réduction linéaire des cotisations patronales (Bureau fédéral du Plan et Banque nationale de Belgique, 2011). Une réduction linéaire a néanmoins un effet favorable mais limité sur l'emploi si les auteurs postulent que les salaires bruts réels n'augmentent pas plus rapidement en présence de la réduction. Une fois que les auteurs relâchent cette hypothèse qu'ils considèrent incompatibles avec les modes de négociation des salaires en place en Belgique, l'impact sur l'emploi est réduit d'un tiers ou de moitié selon le modèle (p.6). Ces études convergent donc sur un deuxième constat :

\section{L'effet positif des réductions de cotisations patronales sur l'emploi s'amenuise à mesure que des hausses de salaires bruts compensent ces réductions.}

Ce constat porte sur la réponse des salaires. Une accélération de la croissance réelle (hors inflation) des salaires bruts «financée» par des allègements de cotisa-

\footnotetext{
${ }^{10}$ Dans le cas espagnol, voir Orsini et al., 2014, qui se basent sur le modèle d'équilibre général dynamique de la Commission européenne (QUEST) et qui recommandent un tax shift ciblé sur les travailleurs faiblement qualifiés.

${ }^{11}$ Ourliac et Nouveau (2012) ainsi que Lehmann et L'Horty (2014) proposent une synthèse de l'expérience et des évaluations françaises. Sur base d'une étude empirique portant sur un panel de pays de l'OCDE, Lehmann et al. (2015) montrent qu'une progressivité accrue du «coin fiscal sur les salaires» (écart entre coût salarial et salaire net) en fonction du salaire net augmente le taux d'emploi et réduit le taux de chômage. La réforme que nous proposons dans cette étude va précisément dans ce sens puisqu'elle diminue le coin fiscal sur les salaires pour les bas salaires et l'augmente (très légèrement) pour les salaires plus élevés.

${ }^{12}$ Ceci n'est pas bien entendu vrai pour tout type de travail peu rémunéré (songeons à certains services aux personnes qui ne se délocalisent ni ne s'automatisent aisément).

${ }^{13}$ Ce constat est mis en évidence par la littérature sur la «polarisation des emplois» (voir par exemple Acemoglu et Autor, 2011).
} 


\section{... Encore mais comment?}

tions patronales en réduit les effets potentiels positifs. ${ }^{14} \mathrm{Ce}$ phénomène est, soulignons-le, compatible avec l'actuelle loi relative à la promotion de l'emploi et à la sauvegarde préventive de la compétitivité datant de 1996 puisque celle-ci porte sur l'évolution du coût salarial et non sur celle des salaires bruts. Or, le risque d'une telle accélération varie selon le ciblage des réductions de cotisations patronales. Si la volonté d'embaucher est stimulée, il importe que la main d'œuvre visée par ces réductions soit disponible en quantité et en qualité. Autrement, tôt ou tard, les salaires subiraient des pressions à la hausse.

Vu le niveau élevé des taux de chômage parmi les moins qualifiés, le volume de main d'œuvre mobilisable est important. Les mécanismes qui freinent une disponibilité effective à travailler doivent continuer à faire l'objet d'une attention particulière. L'intensité de la recherche d'emploi n'est sans doute plus un obstacle important vu la politique d'activation du comportement de recherche appliquée progressivement depuis mi 2004. Il reste dans certains cas la problématique de gains insuffisants en cas de reprise d'emploi mais la dégressivité accrue des allocations de chômage a réduit l'importance de ce type d'obstacle (Conseil Central de l'Economie, 2014). La réduction de cotisations personnelles accordées sous $2.413 €$ bruts par mois (le «bonus à l'emploi») agit dans le même sens. ${ }^{15}$ Enfin, au-delà de l'augmentation des frais professionnels forfaitaires, il y a la promesse du gouvernement fédéral d'octroyer un supplément de revenus nets aux personnes en emploi (pour certaines tranches de revenus il est question de $100 € /$ mois). Si cette mesure prenait la forme d'un crédit d'impôt remboursable, elle bénéficierait aussi aux plus bas salaires. Il reste sans doute des freins notoires à la mobilité géographique induits par le coût excessif du logement dans les sous-régions plus prospères ou par les déficiences de notre système des transports publics.

Là où les qualifications requises, à tort ou à raison, par les entreprises sont relativement élevées, la disponibilité de la main d'œuvre va tôt ou tard freiner les entreprises dans leur propension à embaucher. Ceci s'accompagnera d'une hausse des salaires bruts qui rognera la baisse du coût du travail et au bout du compte la croissance de l'emploi. Plusieurs études belges (Sneessens et al., 2005, Pierrard, 2005, Batyra et Sneessens, 2010) concluent que les réductions de cotisations sociales visant les travailleurs qualifiés n'ont in fine pas d'effet positif sur l'emploi.

Les diverses études mentionnées ci-dessus convergent donc sur l'importance de ne pas utiliser les réductions de cotisations patronales pour financer des hausses réelles de salaires bruts et sur celle de cibler les réductions sur les bas salaires. Par conséquent, même si afficher un taux de cotisations patronales de $25 \%$ peut donner une bonne image dans certains classements internationaux et, par là, probablement influencer certaines décisions de localisation d'investissement, la prise en compte des analyses rappelées ci-dessus invite à cibler l'effort sur le bas de l'échelle des rémunérations si l'augmentation de l'emploi est effectivement la priorité.

Soulignons également qu'accorder un allègement forfaitaire de cotisations sociales patronales (comme c'est le cas encore actuellement à travers le forfait $F$ ) et abaisser

\footnotetext{
${ }^{14}$ Cette affirmation peut surprendre dans la mesure où la hausse des salaires augmente le pouvoir d'achat des travailleurs, tout au moins de ceux qui conservent leur emploi. On en attend généralement des effets bénéfiques sur l'emploi. Mais, une part non négligeable de ce pouvoir d'achat accru se porte sur l'achat de biens importés. En outre, toutes choses égales par ailleurs, la hausse des salaires en Belgique se traduit par un relèvement du coût du travail qui engendre un ensemble d'effets induits dans les entreprises. Tous les modèles macroéconomiques belges concluent qu'une hausse du coût du travail en Belgique a un effet net négatif sur le niveau macroéconomique de l'emploi. Bien qu'improbable vu l'absence d'instrument à ce niveau, une hausse coordonnée des salaires en Europe est un tout autre cas de figure car elle permettrait un renforcement généralisé de la demande finale de biens et services. ${ }^{15} \mathrm{Au}$ troisième trimestre de 2015 , le bonus à l'emploi est une réduction forfaitaire de cotisations sociales personnelles sous un salaire brut mensuel de $1.547 € /$ mois. Ensuite, le bonus décroît linéairement et s'annule lorsque le salaire brut vaut ou dépasse $2.413 €$. Actuellement, le bonus annule le montant des cotisations personnelles si le travailleur gagne mensuellement $1.454 €$, soit un peu moins que le Revenu Minimum Moyen Mensuel Garanti.
} 


\section{... Encore mais comment?}

\section{Une proposition de réforme à l'horizon 2016}

le taux de cotisations sont des réformes d'une toute autre portée. Tout d'abord un allègement forfaitaire a un impact relatif sur le coût du travail qui baisse avec le niveau du salaire brut. Ensuite, les conséquences budgétaires pour la sécurité sociale sont mieux prévisibles lorsque l'allègement est un forfait par travailleur. Seule l'évolution du nombre de salariés introduit de l'incertitude au niveau des dépenses publiques. Quand en revanche on baisse le taux, le manque à gagner par la sécurité sociale dépend à la fois du nombre de salariés et de l'évolution des salaires. Plus ceux-ci augmentent rapidement et plus la baisse de taux a des répercussions budgétaires élevées.

On pourrait penser que l'évolution des salaires bruts dont il a été souvent question ci-dessus peut à long-terme se maîtriser par un gel imposé de ceux-ci ou par une réforme de la loi relative à la promotion de l'emploi et à la sauvegarde préventive de la compétitivité visant à contrôler la croissance des salaires bruts et non plus celle du coût salarial. Même si le dispositif fait l'objet de contrôles réguliers, il y a des raisons de douter que la maitrise de la croissance des salaires bruts soit effective dans la durée. D'abord, cela interfère avec la libre négociation collective bien davantage que la loi actuelle datant de 1996. Ensuite, si l'on impose une même évolution à tous les salaires bruts, on fige les salaires relatifs entre travailleurs de différentes professions ou secteurs. Ceci peut favoriser l'apparition de pénuries de main d'œuvre dans certaines professions ou secteurs. Enfin, le contrôle des salaires bruts peut être contourné, par exemple, par l'octroi d'avantages non salariaux qui échappent à ce contrôle mais qui se répercutent également sur le coût global du travail.

Les résultats d'études scientifiques résumés ci-dessus nous conduisent à formuler la proposition suivante utilisant approximativement les moyens budgétaires prévus par le gouvernement fédéral pour 2016 (1.280 millions). Nous proposons de remplacer la formule existante de la réduction structurelle par une nouvelle formule dans laquelle le niveau de la réduction de cotisations patronales, $R$ (€/mois), dépend du niveau de salaire brut mensuel, $S$ (€/mois), de la manière suivante (dans le cas d'un travailleur à temps plein ${ }^{16}$ ) :

\section{Si $S \leq 1.559: R=0,324 \times S$}

$1.559 €$ est le niveau du RMMMG de janvier 2015 pour un travailleur de 21 ans. A ce niveau, la réduction s'élève à $505 € /$ mois.

\section{Si $1.559<S \leq 2.339: R=505,5-0,45 \times(S-1.559)$}

Le montant de la réduction de cotisations patronales décroît donc linéairement sur la zone de salaires bruts située entre le RMMMG et 1,5 fois le RMMMG ${ }^{17}$. En 2015, environ $35 \%$ des salariés du secteur privé en Belgique gagnent au plus $2.339 € /$ mois.

\section{Si $2.339<S \leq 4.467: R=F=154,2$}

Arrivé à 1,5 fois le $\mathrm{RMMMG}$, on retrouve la réduction forfaitaire mensuelle de $154,2 € /$ mois d'application au $3^{\mathrm{e}}$ trimestre 2015 . Ce forfait est maintenu jusqu'au seuil «haut salaire» actuel, à savoir $4.467 € /$ mois. A ce niveau de salaire, le taux de cotisations patronales après réduction structurelle est inchangé et vaut $29 \%$.

\section{Si $S>4.467: R=0,032 \times S$}

Le montant de la réduction est tel que le taux de $29 \%$ s'applique à tout salaire

\footnotetext{
${ }^{16}$ Pour un travail à temps partiel, il serait logique d'abaisser le montant de la réduction au prorata du temps de travail.

${ }^{17}$ Le niveau précis de ce seuil bas salaires n'est pas guidé par un raisonnement économique précis. Il tient compte de plusieurs considérations : (1) l'existence de minima sectoriels fréquemment supérieurs au RMMMG; (2) le souci de demeurer dans une zone de salaires où la demande de travail est particulièrement sensible à son coût; (3) la volonté de ne pas dépasser l'enveloppe budgétaire prévue par le gouvernement fédéral en 2016.
} 
... Une proposition de réforme à l'horizon 2016 supérieur à $4.467 € /$ mois, si l'on prend 32,4\% comme taux facial de référence.

La figure 3 présente l'allègement de cotisations patronales en vigueur (en vert) et celui proposé ci-dessus (en rouge). La figure 4 illustre le profil du taux (effectif) de cotisations patronales (toujours sous l'hypothèse d'un taux facial de 32,4\%). Notre proposition amènerait le taux effectif moyen de charges patronales de $26 \%$ à $23 \%$. Selon nos estimations, le coût budgétaire annuel du passage du taux effectif actuel au taux effectif sous notre proposition avoisine les 1.270 millions $€$. Notre estimation ne prend pas en compte les économies permises par les effets de notre proposition sur l'emploi en 2016. Sur base d'une estimation rapide de l'impact de cette proposition sur l'emploi, le renforcement de la réduction de cotisations patronales sous $2.339 €$ devrait créer à terme entre 28.000 et 47.000 emplois sous ce seuil de salaire. Cette fourchette est à comprendre à salaires bruts inchangés.

Notre proposition serait d'application en 2016. Elle ne préjuge pas des initiatives à prendre au-delà. Une réflexion s'impose sur le bien-fondé d'accentuer encore les réductions structurelles. Si tel est le cas, le meilleur usage des moyens supplémentaires prévus pour 2018 et 2019 consisterait probablement à atténuer la pente de la relation linéaire au point 2 de notre proposition. Par conséquent, le renforcement de la réduction structurelle se prolongerait au-delà de 1,5 fois le RMMMG. La définition rapide d'une politique pour la période 2016-2019 permettrait aux Régions de prendre de meilleures décisions (coordonnées) sur l'usage des moyens régionalisés relatifs aux réductions de cotisations sur les «groupes cibles» et aux activations d'allocations de chômage.
Figure 3. Niveau de la réduction de cotisations sociales patronales en fonction du salaire mensuel brut entre le RMMMG à 21 ans et $6000 € /$ mois. En vert la situation au $3^{\mathrm{e}}$ trimestre de 2015. En rouge, notre proposition pour 2016. La ligne verticale en pointillés se situe à 1,5 fois le RMMMG.

Figure 4. Taux de cotisations sociales patronales en fonction du salaire mensuel brut entre le RMMMG à 21 ans et $6000 € /$ mois. En vert la situation au $3^{\mathrm{e}}$ trimestre de 2015 . En rouge, notre proposition pour 2016. La ligne verticale en pointillés se situe à 1,5 fois le RMMMG.

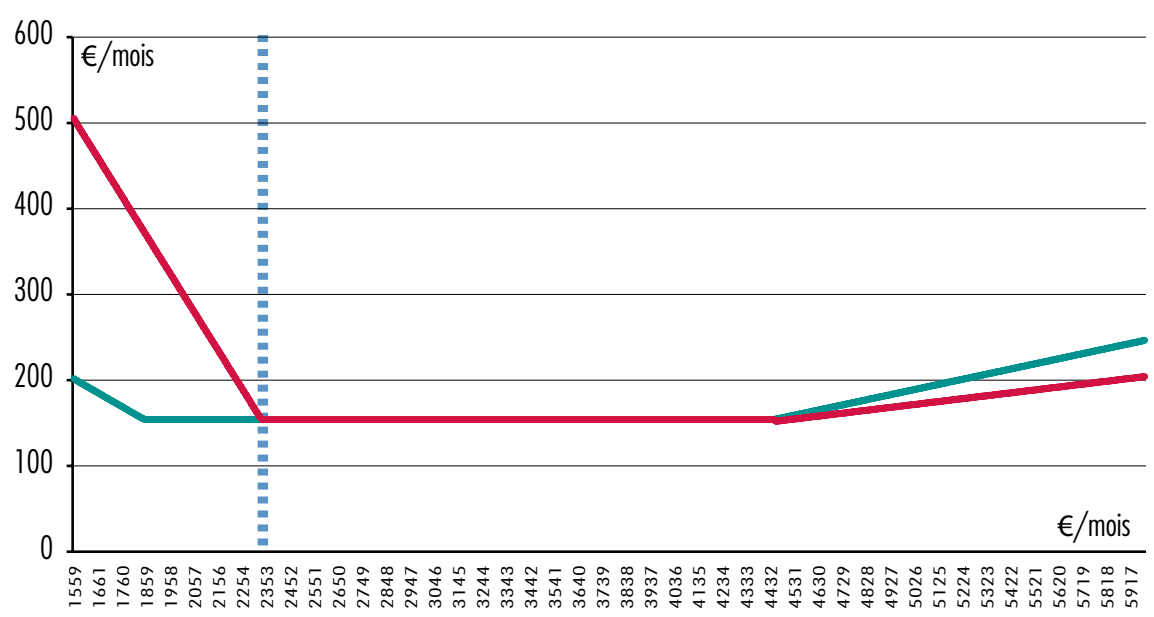




\section{De quelques critiques souvent entendues concernant les réductions sur les bas salaires}

Concentrer les réductions de cotisations patronales sur les bas salaires est une proposition déjà souvent entendue. Elle a été critiquée sous divers angles que nous énumérons ici en indiquant nos éléments de réponse.

1. «L'allègement du coût du travail au bas de l'échelle des salaires est un combat perdu d'avance parce que la concurrence des pays à bas salaires est inexorable». Même si l'on tient compte des différences de productivité du travail, la réalité de cette concurrence est incontournable. Mais, comme le montre l'expérience chinoise, il vient un moment où, d'une manière ou d'une autre, ces pays procèdent à une hausse des rémunérations. On ne peut que s'en réjouir pour la population de ces pays et nos travailleurs peu qualifiés. Il faut aussi rappeler que, pour les entreprises belges, la concurrence internationale s'exerce d'abord entre pays européens et qu'à ce niveau, une absence de déséquilibre entre les coûts salariaux par unité produite, loin d'être acquise, demeure accessible.

2. «La notion de bas salaire est une pauvre approximation de celle de peu qualifié». Nous sommes bien conscients de cette limite inhérente à la collecte d'informations par la sécurité sociale. Toutefois, comme le souligne Cockx (2013, p. 12), «l'évolution salariale doit plus ou moins correspondre à celle de la productivité». Dans un régime où les réductions de charge sont ciblées sur les bas salaires, les réductions diminuent progressivement à mesure que le salaire augmente pour éviter les effets de seuil (comme une disparition brutale de la réduction quand le travailleur a un diplôme supérieur à un niveau de référence, disparition qui peut introduire des inégalités injustifiées entre travailleurs). «La diminution sera donc beaucoup plus conforme à la croissance de la productivité que n'importe quel autre ciblage». En raison de l'évolution des salaires avec l'ancienneté dans l'entreprise ou sur le marché du travail, un salarié peu qualifié âgé gagne en outre typiquement davantage qu'un jeune. La prise en compte de l'âge relève à présent de la compétence des Régions.

3. «La stimulation d'une demande de travail pour les peu qualifiés inciterait à la déqualification des travailleurs qui ont des compétences qui surpassent celles requises pour les emplois à bas salaires. Cette concurrence exacerbée sur les emplois à bas salaires viderait de son intérêt la mesure de réduction de cotisations ciblée sur les bas salaires ». Il est vrai que cette possibilité de déqualification atténue l'impact positif des allègements sur les travailleurs moins qualifiés visés par la mesure, mais il est faux que la mesure perd tout intérêt (Pierrard, 2005, Batyra et Sneessens, 2010, Belan et al. 2010). Notons par ailleurs que s'il y a un problème d'acceptation trop peu fréquente des offres d'emploi en bas de l'échelle des salaires, cette déqualification atténue les tensions salariales qui risqueraient de survenir si on stimule la demande de travail à ce niveau.

4. «L'abaissement du coût du travail en bas de l'échelle des salaires entraînera des substitutions dans l'entreprise en défaveur de l'emploi mieux rémunéré». Cet effet est à prendre en compte. Mais dans la mesure où la réduction des cotisations patronales sur les bas salaires décroît avec le niveau de ceux-ci (voir figures 1 et 3 ) et dans la mesure où les individus les plus proches (ici en termes de salaire) sont les plus substituables, l'effet de substitution entre types de main d'œuvre est à relativiser fortement.

5. «Le ciblage des réductions de cotisations sur les bas salaires crée un piège à bas salaire». Lorsque la réduction de cotisations décroît avec le niveau du salaire, l'octroi d'une augmentation de salaire brut s'accompagne d'une baisse de la réduction octroyée. En quelque sorte, l'octroi d'une augmentation de salaire brut est «taxée». Dans notre proposition, entre le RMMMG et 1,5 fois le RMMMG, chaque euro supplémentaire de salaire brut entraîne une diminution de la réduction $R$ de 45 centimes (contre 16 dans la zone bas 


\author{
... De quelques critiques souvent \\ entendues concernant les \\ réductions sur les bas salaires
}

salaire actuelle). Même si le caractère dissuasif de cette «taxation» implicite n'a pas encore été étudié en Belgique ${ }^{18}$, l'argument a de la pertinence, d'autant que les allègements de cotisations personnelles (le bonus à l'emploi) agissent dans le même sens. En France, où l'allègement de cotisations sociales patronales est maximal au SMIC (le salaire minimum) y valant jusqu'à 28 points de pourcentage depuis 2008 et s'annule à 1,6 fois le SMIC (L'Horty et Lehmann 2014), quelques études ont porté sur la question. Ourliac et Nouveau (2012) les synthétisent et concluent (p. 20) : «Au final, les études menées sur l'impact des allègements sur la distribution des salaires et la dynamique salariale ne semblent pas confirmer l'existence de 'trappes à bas salaires' significatives, même si elles ne permettent pas non plus de l'écarter totalement».

6. «Le développement d'emplois peu productifs réduirait encore les gains de productivité moyenne du travail en Belgique». Un tel effet est indéniable. La hausse de la productivité n'est cependant pas une fin en soi. ${ }^{19}$

7. «Le développement d'emplois à relativement bas salaires serait synonyme d'emplois de piètre qualité». Soulignons que le ciblage des cotisations sur les bas salaires s'effectue sans remise en question ni du salaire minimum (RMMMG), ni de la négociation collective. Par conséquent, cette critique apparaît peu fondée. C'est en particulier vrai si la mobilité salariale ascendante n'est pas mise en cause (voir critique 5 ci-dessus).

8. «La stimulation d'une demande de travail pour les peu qualifiés n'inciterait pas ces derniers à se former». Le rendement de l'éducation demeure substantiel en Belgique (salaires supérieurs, avantages en nature, risque de chômage atténué). Une amélioration des perspectives d'embauche pour les travailleurs moins qualifiés ne devrait donc pas inverser la tendance généralisée à l'augmentation des niveaux d'étude et de formation.

9. «Les allègements de cotisations sociales patronales seraient un chèque en blanc au bénéfice des employeurs». Il faudrait accompagner ces allègements de contrôles visant à assurer le «bon usage» du «chèque». Il n'y a aucune objection de principe à associer des allègements à un contrôle. Mais, il faut bien prendre garde aux effets pervers de ce contrôle et à l'applicabilité du contrôle envisagé. Ainsi, on pourrait imposer que l'allègement de cotisations ne soit payé après coup qu'aux entreprises dont la variation (disons, annuelle) du volume de l'emploi a dépassé un seuil. Fixer ce seuil à l'avance apparaît peu sensé vu les incertitudes macroéconomiques de court terme. Même si le seuil doit donc être défini rétrospectivement, ce type de condition n'est a priori pas absurde. Il faut néanmoins s'interroger sur les conséquences de cette condition sur l'attitude des employeurs et in fine sur l'emploi. Au-delà de chocs macroéconomiques et sectoriels qui pourraient être pris en compte dans le seuil, les entreprises subissent des chocs qui leur sont spécifiques et inobservables ou invérifiables par une autorité publique. Ces chocs spécifiques peuvent être tantôt positifs et rendre le dépassement du seuil aisé, tantôt négatifs et produire l'effet opposé. Que nous apprend réellement l'évolution de l'emploi d'une entreprise particulière au sujet du bon ou du mauvais usage d'un allègement de cotisations ? Bien moins qu'il n'y paraît. En outre, ces chocs spécifiques

\footnotetext{
${ }^{18}$ On ne dispose que de l'évidence indirecte suivante. Si l'on s'intéresse à la sortie du premier quintile de rémunérations (les $20 \%$ les plus bas de la distribution des salaires), selon les calculs de Ries (2013), la Belgique surpasse ses voisins, en ce sens que, d'une année à l'autre, $29 \%$ des Belges opèrent une telle transition contre $27 \%$ en France, $26 \%$ au Royaume-Uni, $19 \%$ en Allemagne et seulement $13 \%$ aux Pays-Bas (la moyenne au sein de l'Union des 27 étant de $25 \%$ ).

${ }^{19}$ A cet égard, il est intéressant de noter que Lehmann et al. (2015) montrent qu'une augmentation de la progressivité des prélèvements obligatoires a un effet positif sur l'emploi du même ordre de grandeur que l'effet négatif sur la productivité du travail. Par conséquent, l'effet net d'une hausse de la productivité sur la production totale est à peu près nul.
} 


\author{
... De quelques critiques souvent \\ entendues concernant les \\ réductions sur les bas salaires
}

aléatoires introduisent de l'incertitude dans l'octroi de l'allègement. Comment les décisions d'embauche et de licenciement seront-elles prises face à un allègement de coût aléatoire ? Sans donc fermer la porte à l'imposition de conditions à l'octroi des allègements, nous plaidons pour que leur introduction éventuelle prenne très sérieusement en compte la réalité de la prise de décision au sein des entreprises.

10. «Le ciblage sur les bas salaires oublie les secteurs d'avenir». Il est clair que l'allègement ciblé sur les bas salaires ne touche pas tous les secteurs de la même manière (Van der Linden 2005). Les secteurs les moins concernés sont ceux où les bas salaires sont rares. Il s'agit typiquement de certains secteurs industriels et de plus grandes entreprises. Rappelons que réduire les cotisations sociales patronales sur les salaires élevés est beaucoup plus coûteux. Dans le cas de secteurs ouverts au commerce international, un ciblage sectoriel enfreindrait en outre les règles de saine concurrence. Notons aussi que le gouvernement Michel prévoit de renforcer l'exemption de précompte sur le travail en continu et en équipe à raison de 450 millions $€$. Ce faisant, il vise essentiellement un certain nombre de secteurs industriels.

11. «Le ciblage sur les bas salaires oublie la problématique de la fuite des cerveaux». Les personnes à (très) hautes qualifications peuvent, par l'innovation qu'elles rendent possibles ou leurs talents entrepreneuriaux, jouer un rôle central dans le développement de l'emploi. Le poids des prélèvements obligatoires sur les travailleurs (très) qualifiés devrait être réduit afin justement de pouvoir leur accorder des rémunérations plus élevées et ainsi d'éviter leur départ pour un pays étranger. La mobilité internationale des travailleurs (très) qualifiés est indéniable. L'instauration de régimes dérogatoires temporaires sur les prélèvements obligatoires de ces travailleurs apparaît efficace pour les maintenir au pays ou attirer des étrangers (voir Kleven et al., 2013, pour une analyse fine du cas danois). Il est cependant plausible que les facteurs qui pèsent dans les décisions de mobilité internationale sont, outre la rémunération nette, le coût de la vie, la qualité des soins de santé et de l'enseignement, etc. En outre, s'il s'agit de réfléchir à un «package attractif» pour certaines catégories de travailleurs très qualifiés, il n'est nullement besoin de déplacer des masses budgétaires pour alléger les cotisations sociales patronales de l'ensemble des travailleurs à rémunération (très) élevée étant donné les évidences rappelées plus haut quant aux effets nets sur l'emploi.

La mesure dite de «tax shift» du gouvernement fédéral fait couler beaucoup d'encre. Nous l'avons abordée sous l'angle fondamental du ciblage de la réduction du coût du travail. L'enveloppe budgétaire prévue ne permettra pas de financer à la fois une réduction généralisée des cotisations patronales (le passage à un taux facial effectif de $25 \%$ ) et un renforcement significatif de la réduction structurelle sur les bas salaires. Des choix importants s'imposent quant à l'affectation de cette enveloppe budgétaire. Considérant que la création d'emplois est la priorité, nous avons rappelé que le ciblage sur les bas salaires s'impose. Une autre forme de ciblage aurait en effet un impact nul ou très limité sur l'emploi.

Nous avons formulé une proposition à l'horizon 2016 qui s'inscrit dans l'enveloppe prévue par le gouvernement fédéral pour cette année (1.280 millions $€$ ). Cette proposition fait disparaître le maximum possible de cotisations patronales $(32,4$ points de pourcentage) au niveau du revenu minimum moyen garanti en Belgique (1.559€ bruts par mois à 21 ans). A ce niveau, la réduction de cotisations patronales représente plus de $500 € /$ mois. Ensuite, la baisse de charges se réduit linéairement jusqu'à 1,5 fois le revenu minimum $(2.339 €)$ où elle atteint la réduction forfaitaire de cotisations patronales en vigueur actuellement (154 €/mois). 
... Conclusion

Muriel Dejemeppe est professeure d'économie à l'UCL et chercheuse à l'IRES.

Bruno Van der Linden est maître de recherche du Fonds National de la Recherche Scientifique - FNRS, professeur d'économie à l'UCL et chercheur à l'IRES.
Cette réduction forfaitaire inchangée s'applique jusqu'au seuil «haut salaire» actuel de $4.467 € /$ mois. A ce niveau, le taux de cotisations patronales s'élève à $29 \%$. Ce taux s'applique au-delà du seuil «haut salaire». Notre proposition amènerait le taux effectif moyen de charges patronales de $26 \%$ à $23 \%$, si l'on prend $32,4 \%$ comme taux facial de référence.

Le renforcement de la réduction de cotisations patronales sous $2.339 €$ devrait créer ou sauvegarder à terme entre 28.000 et 47.000 emplois sous ce seuil de salaire.

Ce texte n'a pas abordé l'importante question du financement d'une réduction renforcée des cotisations patronales sur les travailleurs à bas salaires. Avec le ciblage que nous proposons, l'effet des réductions de cotisations sociales patronales sur l'emploi est maximal. Plus les retombées sur l'emploi sont favorables, moins la question du financement alternatif de la sécurité sociale est aiguë. Ce financement devrait être étendu en choisissant les instruments qui nuisent le moins à l'emploi. Les outils de modélisation développés au Bureau fédéral du Plan et à la Banque nationale de Belgique fournissent à cet égard des informations incontournables (voir par exemple Bassilière et al., 2005).

Compte tenu des effets limités sur l'emploi d'une réduction de charges patronales sur les salaires moyens et élevés, une proposition qui amènerait le taux effectif de cotisations patronales à $25 \%$ engendrerait un besoin de financement alternatif de la sécurité sociale nettement supérieur.

\section{Références}

Acemoglu D. et D. Autor (2011). "Skills, tasks and technologies: Implications for employment and earnings", chapter 12, in O. Ashenfelter and D. Card, Handbook of Labor Economics, vol 4A, North-Holland.

Bassilière D., F. Bossier, I. Bracke, I. Lebrun, L. Masure et P. Stockman (2005). Variantes de réduction des cotisations sociales et modalités de financement alternatif, Planning Paper 97, Bureau fédéral du Plan, Bruxelles, janvier.

Batyra A. et H. Sneessens (2010). "Selective reductions in labor taxation: Labor market adjustments and macroeconomic performance", Journal of Policy Modelling, 32, p. 531-543.

Belan P., M. Carré et S. Gregoir (2010). "Subsidizing low-skilled jobs in a dual labor market", Labour Economics, 17, 776-788.

Bureau fédéral du Plan et Banque nationale de Belgique (2011). Réductions des cotisations sociales et modalités de financement alternatif. 27 avril 2011. 
... Références
Cockx, B., H. Sneessens, B. Van der Linden, A. Batyra, G. Cardullo, M. Dejemeppe, M. Denni, C. Goebel et E. Moreno (2005), Evaluations micro- et macroéconomiques des allègements de la parafiscalité en Belgique, Politique scientifique fédérale et Academia Press, Gent.

Cockx B. (en collaboration avec Muriel Dejemeppe) (2005). "Evaluation microéconométrique des allègements structurels et bas salaires pour une population de jeunes défavorisés", chapitre 3, in Cockx, B., H. Sneessens, B. Van der Linden, A. Batyra, G. Cardullo, M. Dejemeppe, M. Denni, C. Goebel et E. Moreno (2005), Evaluations micro- et macro-économiques des allègements de la parafiscalité en Belgique, Politique scientifique fédérale et Academia Press, Gent. http://www.belspo.be/belspo/fedra/proj.asp?1=fr\&COD=SO/10/073

Cockx B. (2013). "Le chômage des jeunes en Belgique. Diagnostic et remèdes clés”, Regards économiques, 108, décembre, IRES, Louvain-la-Neuve.

Conseil Central de l'Economie (2014). La dégressivité renforcée des allocations de chômage, Document CCE 2014 - 0264, Conseil Central de l'Economie, Bruxelles.

Conseil Central de l'Economie (2015). Annexes au Rapport technique, Document CCE 2015 - 1150, Conseil Central de l'Economie, Bruxelles.

Falk A., A. Kuhn et J. Zweimüller (2011). "Unemployment and right-wing extremist crime", Scandinavian Journal of Economics, 113(2), 260-285.

Goos M. (2007). "The impact of payroll tax subsidies on employment and wages: A Natural Experiment Using Firm Level Data”, LICOS Discussion Papers 17807, LICOS - Centre for Institutions and Economic Performance, K.U.Leuven. Une synthèse a été publiée par Over.Werk, 2007, $\mathrm{n}^{\circ} 1$.

Kleven H.J., C. Landais, E. Saez et E. Schultz (2014). "Migration and wage effects of taxing top earners: Evidence from the foreigners' tax scheme in Denmark", Quarterly Journal of Economics, 129(1), 333-378.

Lehmann E. et Y. L’Horty (2014). "Renforcer la progressivité des prélèvements sociaux", Revue française d'économie, XXIX, 25-61.

Lehmann E., C. Lucifora, S. Moriconi et B. Van der Linden (2015). "Beyond the Labour Income Tax Wedge: The Unemployment-Reducing Effect of Tax Progressivity", à paraître dans International Tax and Public Finance.

Orsini K., M. Burgert, O. Grevesmühl et M. Suardi (2014). “Assessing the impact of a revenue-neutral tax shift away from labour income in Spain". ECFIN Country Focus, 11(5).

http://ec.europa.eu/economy_finance/publications/country_focus/2014/pdf/cf_vol11_issue5_en.pdf

Ourliac B. et C. Nouveau (2012). "Les allègements de cotisations sociales patronales sur les bas salaires en France de 1993 à 2009", Direction de l'animation de la recherche, des études et des statistiques (DARES), Document d'études 169, Février.

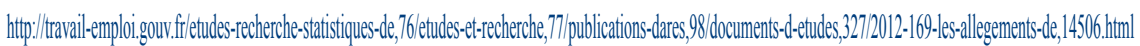

Pierrard O. (2005). "Impacts of selective reductions in labor taxation", Applied Economics Quarterly, 51(1), 49-82.

Ries J. (2013). Patterns of low-wage employment and related indicators in Europe. Luxembourg : Working paper 66 du STATEC, juin, Luxembourg.

Rycx F., Y. Saks et I. Tojerow (2015). "Does education raise productivity and wages equally? The moderating roles of age, gender and industry", Working Paper Dulbés 15-02.

http://difusion.ulb.ac.be/vufind/Record/ULB-IPOT:oai:dipot.ulb.ac.be:2013/199356/Holdings 
... Références
Sneessens H., A. Batyra, M. Denni et E. Moreno (2005). "Evaluation macro-économique", chapitre 5, in Cockx, B., H. Sneessens, B. Van der Linden, A. Batyra, G. Cardullo, M. Dejemeppe, M. Denni, C. Goebel et E. Moreno, Evaluations micro- et macro-économiques des allègements de la parafiscalité en Belgique, Politique scientifique fédérale et Academia Press, Gent. http://www.belspo.be/belspo/fedra/proj.asp?l=fr\&COD=SO/10/073

Van der Linden B. (2005). "Une proposition de renforcement des allègements de cotisations sociales patronales sur les bas salaires", Regards économiques, 34, octobre, IRES, Louvain-la-Neuve.
Directeur de la publication :

Vincent Bodart

Rédactrice en chef :

Muriel Dejemeppe

Comité de rédaction : Paul Belleflamme,

Vincent Bodart, Muriel Dejemeppe, Frédéric

Docquier, Jean Hindriks, Marthe Nyssens,

William Parienté, Mikael Petitjean

Secrétariat \& logistique : Anne Davister

Graphiste : Dominos
Regards Économiques IRES-UCL

Place Montesquieu, 3

B1348 Louvain-la-Neuve

www.regards-economiques.be

regard-ires@uclouvain.be

tél. 010/473426 